\title{
LETTERS
}

doi:10.1017/S1041610207006400

\section{Is there a shift of burden from geriatric medicine services to old age psychiatry services?}

The introduction of the Community Care (Delayed Discharge) Act 2003 in England led to a reduction in the mean and median length of stay of patients admitted to geriatric medicine (GM) units despite an increase in the number of admissions (Shah, 2007) because it imposed financial penalties on local authorities for delays in the discharge of patients. However, mean and median length of stay of patients admitted to old age psychiatry (OAP) units increased, along with the number of bed days utilized, despite a reduction in the number of admissions (Shah, 2007). This raised concerns that community care budgets may have been moved from OAP services to GM services because there were no financial penalties for delays in discharging patients from OAP units. However, closer examination of the data suggested that some of the observed changes began to occur before the Community Care Act 2003 was implemented, particularly in OAP services. This raised the possibility that other governmental initiatives may have also shifted the burden from GM services to OAP services prior to the implementation of this Act. This shift in burden from GM services to OAP services has been formally examined and is reported here.

Data on the number of finished inpatient episodes, mean and median length of stay, and the number of bed days for patients admitted to GM and OAP services in England were ascertained from nationally collected data (Hospital Episode Statistics - www.hesonline.nhs.uk/Ease/servlet/ContentServer?siteID= 1937\&CategoryID=192) for the eight financial years between 1998/1999 and 2005/2006. Each of these eight years was numerically coded from 1 to 8 . Trends over time for all the measured variables were examined by correlating these numerical codes and the measured variables in GM and OAP using Spearman's correlation coefficient $(\rho)$. Also, the relationship between GM and OAP services for all the measured variables was examined using Spearman's correlation coefficient. Furthermore, a trend over time for the combined total of bed days for both GM and OAP services was examined to find evidence supporting the hypothesis that the number of bed days in the combined GM and OAP services remained constant over the eight-year period.

In GM services, there was a decline in the median length of stay $(\rho=-0.76$, $\mathrm{p}=0.027)$ and an increase in the number of finished inpatient episodes $(\rho=+0.79, \mathrm{p}=0.021)$ over the eight-year period; there was no change over time in the mean length of stay and bed days. In OAP services, there was an increase in the mean $(\rho=+0.91, \mathrm{p}=0.002)$ and median $(\rho=+0.99$, $\mathrm{p}<0.0001)$ length of stay, and in bed days $(\rho=+0.93, \mathrm{p}<0.0001)$, and a decline in the number of finished inpatient episodes $(\rho=-0.95, \mathrm{p}<0.0001)$ 
over the eight-year period. There were significant negative correlations for finished inpatient episodes $(\rho=-0.74, \mathrm{p}=0.037)$ and median length of stay $(\rho=-0.77, \mathrm{p}=0.024)$ between GM and OAP services, and the combined total number of bed days for GM and OAP services did not change over this period.

The significant increase in the number of finished episodes, and decrease in the median length of stay in GM services and the opposite trend in OAP services over the eight-year period, the negative correlations for finished inpatients episodes and median length of stay between GM and OAP services, and an absence of a change in the combined total number of bed days for GM and OAP suggest that the burden of inpatient service provision may have shifted from GM services to OAP services. It is possible that various governmental initiatives, including the Community Care Act 2003 and the National Service Framework for Older People, may have simply shifted the burden from GM services to OAP services. Therefore, patients who were previously admitted to GM services from the community may have subsequently been admitted to OAP services in recent years. For example, the development of intermediate care services may have allowed GM services to avoid admissions and discharge patients earlier with the aim of maintaining them in the community. However, it is also possible that some of these patients may not have remained in the community, and instead they may have been admitted to OAP units, and this is supported by the combined total number of bed days for both GM and OAP services remaining constant over the eight years. Many OAP services have not been involved locally in the development of intermediate care services and many intermediate care services specifically exclude patients with mental illness (Baldwin, 2003). This suggests that OAP services may not have been beneficiaries of intermediate care services. The hypothesis that governmental initiatives may simply shift the burden from one service to another without reducing the overall burden for all services requires rigorous testing in multi-center individual-level studies otherwise elderly mentally ill patients will continue to experience discrimination.

\section{References}

Baldwin, R. C. (2003). National Service Framework for Older People. Psychiatric Bulletin, 27, 121-122.

Shah, A. K. (2007). The impact of the Community Care (Delayed Discharge) Act 2003 on the length of stay and bed occupancy in old age psychiatry units in England. International fournal of Geriatric Psychiatry, 22, 1164-1165.

\section{AJIT SHAH}

Professor of Ageing, West London Mental Health NHS Trust, Middlesex, U.K. Email: ajit.shah@wlmht.nhs.uk 